\title{
Radiation-Driven Disk Winds
}

\author{
Janet E. Drew, Daniel Proga, and René D. Oudmaijer \\ Department of Physics, Blackett Laboratory, \\ Imperial College of Science Technology \& Medicine, \\ Prince Consort Road, London SW7 2BZ, United Kingdom
}

Abstract. Recent work on numerical modelling of radiation-driven disk winds is summarised and discussed in the context of extreme B emission line stars.

\section{Motivation}

Bright disks are found in many astrophysical settings: around young stars, in AGN, in interacting binaries and, indeed, they probably occur in the extreme B emission line stars typically classified as B[e] stars. Such disks may shine either by virtue of radiative energy generation due to accretion, or as a consequence of reprocessing light from a luminous central object that falls upon them. In either case, radiative momentum is available to direct a flow away from the disk surface. Just as luminous spherical stars can power significant mass loss via radiation pressure, provided the stellar luminosity exceeds $\sim 0.001 L_{E}$ (where $L_{E}$ is the Eddington luminosity), so too may a circumstellar disk. The challenge, that has only recently been met, is to demonstrate how this is realised within the more complex disk geometry.

Our work in this area is now described in three published papers: namely, Proga, Stone \& Drew (1998); Drew, Proga \& Stone (1998) and Oudmaijer et al. (1998). In this short contribution we draw attention to those aspects of this work most relevant to the study of luminous hot stars. It complements the efforts in recent years to explain the origin of circumstellar disks in terms of either wind compression (see Bjorkman, this volume) or 'bistability' (see Lamers this volume). Our philosophy is that we do not attempt at this stage to explain both circumstellar disks and the flows associated with them instead we assume that an optically-thick, geometrically-thin disk exists and then numerically model the radiation-driven outflow that may arise from it. Our models will accordingly apply directly where circumstellar disks are nearly Keplerian, and a product of non-radiative dynamical processes (e.g. accretion, or excretion as a result of pulsation or prior stellar dynamical evolution).

\section{Method}

Over the past decade or so, there have been several onslaughts on the disk wind problem. The majority have been semi-analytic treatments (e.g. Vitello 
\& Shlosman 1988, Murray et al. 1995) that achieved little predictive power. Our approach owes most to the pioneering work of Icke (1981). We have adopted a numerical approach similar to his in order that approximation of the truly multi-dimensional character of the problem be kept to a minimum. We have moved on from Icke's electron-scattering models in that we treat line-driving using a CAK-like parameterisation of the radiative acceleration (see Proga et al. 1998 for details). The hydrodynamical code we have used is a version of ZEUS-2D (Stone \& Norman 1992) in which the third spatial dimension is accounted for by axisymmetry and conservation of angular momentum.

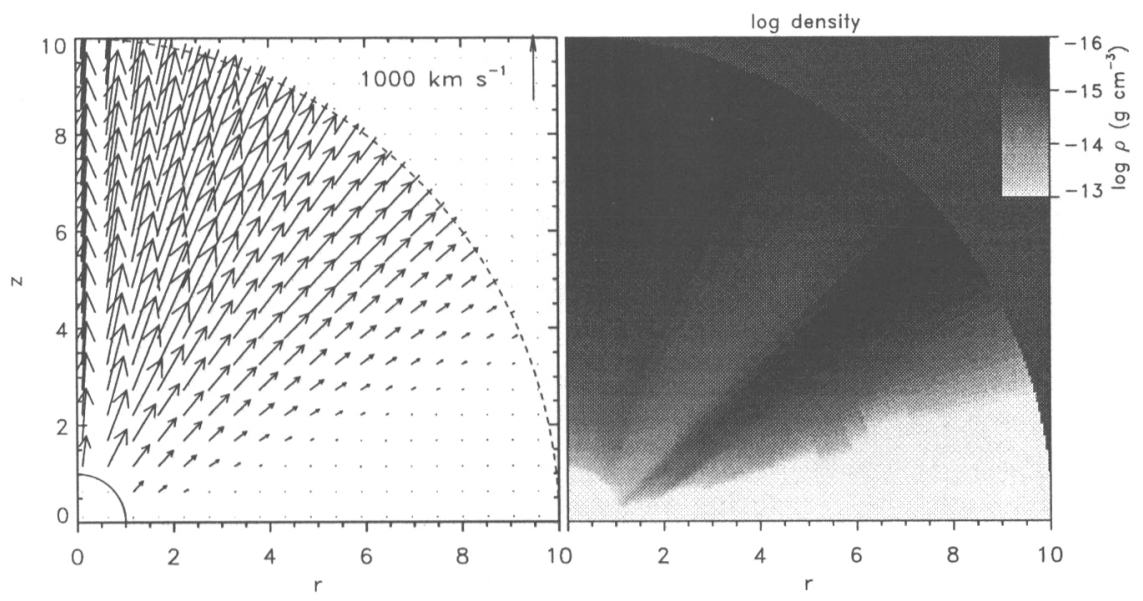

Fig. 1. The velocity field (left) and grey-scale density map (right) for a model of an evolved B star with a Keplerian circumstellar disk, described in the text. The rotational axis of the disk is along the left hand side vertical frame in each panel, while the disk midplane is along the bottom horizontal frame. The vertical and horizontal spatial scales are both expressed in units of the stellar radius. To emphasise the density changes in the outflow, we set the greyscale upper limit to saturate at $10^{-13} \mathrm{~g} \mathrm{~cm}^{-3}$. Note that the left panel omits the rotational component of the velocity.

In our more recent models, calculated for two early B-star examples (Drew et al. 1998, Oudmaijer et al. 1998), the surfaces of both the circumstellar disk and star are included in the calculations as mass-losing and radiant boundaries. Purely to ease gridding of the computational domain, the star is assumed to be non-rotating (this is a convenient rather than a shaping feature of the models). However, an assumption that is essential to the outcome is that the circumstellar disk is optically-thick - only because of this is the disk able to reprocess the starlight incident upon it and become a dynamically significant source of radiation. Gayley (this volume) presents an independent 
discussion of how the CAK force responds to an irradiated surface. In advance of the appropriate full disk atmosphere calculations, we make the simplest assumption that the disk re-emits starlight falling upon it isotropically (cf. Kenyon \& Hartmann 1987).

\section{Results}

Figure 1 shows the calculated density and velocity field obtained for the case of mass loss driven from a disk and evolved B star (taken from Oudmaijer et al. 1998). Note that the disk surface flux due to reprocessing of starlight falls off as $R^{-3}$ in essentially the same manner as in a self-luminous accretion disk. This trend may be compared with the $R^{-2}$ overall decline in gravity. Hence regardless of whether the disk shines by its own light or starlight, radiation is most effective at driving mass loss from the inner disk. This shows up in the illustrated model as a peak in mass flux $\left(\rho v_{r}\right)$ along streamlines emanating from within two stellar radii at a polar angle of $70^{\circ}$.

In brief, we find that a radiation-driven circumstellar disk wind is typically a slower, denser flow than the flow originating directly from the stellar surface. This difference can be seen as a consequence of the changed inner boundary condition - the stellar wind component is launched from the subcritically rotating photosphere, while the disk wind is launched from Keplerian orbits in the equatorial plane beyond the stellar surface. A new result, that is at once both reassuring and very striking, is that the integrated disk wind mass loss rate can be shown to be within a factor of two of the CAK prediction for its spherically-symmetric equivalent (Proga, in preparation). Observationally the significance of such disk winds would be that low equatorial wind speeds can be easily explained, as might high pole/equator density contrast (depending on how the details of spectral line transfer work out). The results presented in Oudmaijer et al. (1998) are, in effect, a possible numerical realisation of Zickgraf et al.'s (1985) conceptual model for B[e] stars. A next step for us will be to discover whether these models can be converted into synthetic line profiles reminiscent of real systems. Work is in progress.

\section{References}

Drew J.E., Proga D., Stone J.M. 1998, MNRAS, 296, L6

Icke V., 1981, ApJ, 247, 152

Kenyon S.J., Hartmann L. 1987, ApJ 323, 714

Murray N., Chiang J., Grossman S. A., Voit G. M., 1995, ApJ, 451, 498

Oudmaijer R. D., Proga D., Drew J. E., de Winter D., 1998, MNRAS, in press

Proga D., Stone J. M., Drew J.E. 1998, MNRAS, 295, 595

Stone J. M., Norman M. L., 1992, ApJS, 80, 753

Vitello P. A. J., Shlosman I., 1988, ApJ, 327, 680

Zickgraf F.-J., Wolf B., Stahl O., Leitherer C., Klare G. 1985, A\&A 143, 421 


\section{Discussion}

A. Feldmeier: I am wondering why your model prefers a wind from inner disk regions. For outer disk regions, the increase in the flux with height due to illumination by inner hot regions should be more pronounced, and should help the gas to overcome the linear increase of gravity with height. Furthermore, both $g$ and $\mathbf{F}_{\text {disk }}$ go essentially as $\sim 1 / \mathrm{r}^{2}$.

D. Proga: In the ideal $\alpha$-disk considered in the models, the effective temperature declines as (disk radius) ${ }^{-3 / 4}$. Hence the luminosity per unit area falls off as the cube of the radius - more quickly, therefore, than gravity. Illumination by inner hotter regions is not enough of an effect to overcome this.

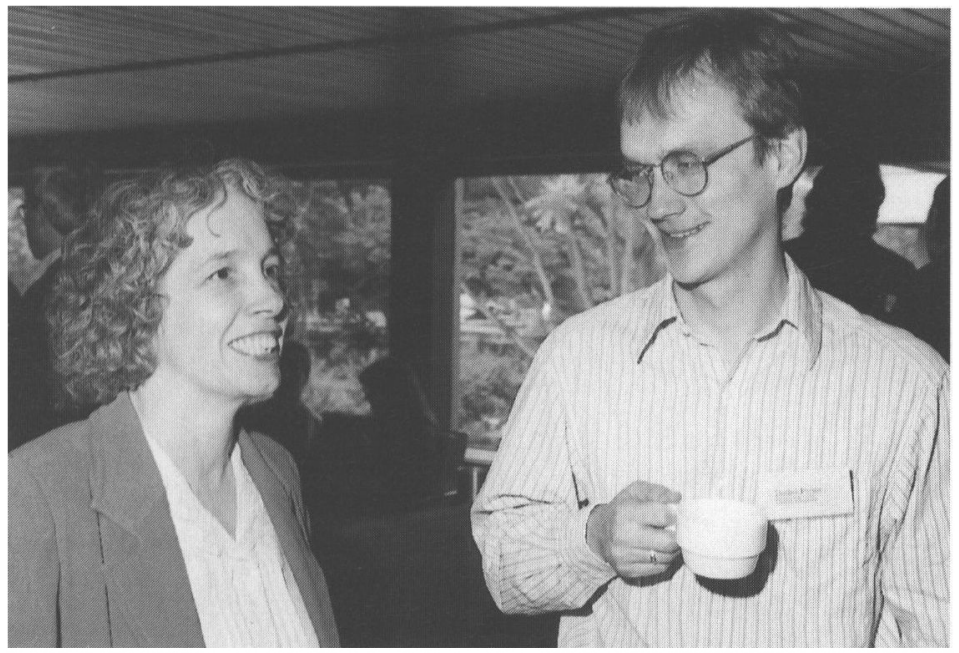

Janet Drew and Daniel Proga 